IMAFF- $95 / 37$

NIKHEF-95-063

hep-th/9511208

\title{
The Other Spectral Flow
}

\author{
Beatriz Gato-Rivera $^{a, b}$ and Jose Ignacio Rosado ${ }^{a}$ \\ ${ }^{a}$ Instituto de Matemáticas y Física Fundamental, CSIC, \\ Serrano 123, Madrid 28006, Spain 7 \\ ${ }^{b}$ NIKHEF-H Kruislaan 409, NL-1098 SJ Amsterdam, The Netherlands
}

\begin{abstract}
Recently we showed that the spectral flow acting on the $\mathrm{N}=2$ twisted topological theories gives rise to a topological algebra automorphism. Here we point out that the untwisting of that automorphism leads to a spectral flow on the untwisted $\mathrm{N}=2$ superconformal algebra which is different from the usual one. This "other" spectral flow does not interpolate between the chiral ring and the antichiral ring. In particular it maps the chiral ring into the chiral ring and the antichiral ring into the antichiral ring. We discuss the similarities and differences between both spectral flows. We also analyze their action on null states.
\end{abstract}

November 1995

*e-mail addresses: bgato, jirs @pinar1.csic.es 


\section{Introduction}

In a recent paper [1] we analyzed the action of the spectral flow on the topological theories obtained by twisting a $\mathrm{N}=2$ superconformal theory. We found that the spectral flow interpolates between the two twisted theories corresponding to a given superconformal one in the following way

$$
\begin{aligned}
\mathcal{U}_{1} \mathcal{L}_{m}^{(2)} \mathcal{U}_{1}^{-1} & =\mathcal{L}_{m}^{(1)}-m \mathcal{H}_{m}^{(1)} \\
\mathcal{U}_{1} \mathcal{H}_{m}^{(2)} \mathcal{U}_{1}^{-1} & =-\mathcal{H}_{m}^{(1)}-\frac{\mathrm{c}}{3} \delta_{m, 0} \\
\mathcal{U}_{1} \mathcal{Q}_{m}^{(2)} \mathcal{U}_{1}^{-1} & =\mathcal{G}_{m}^{(1)} \\
\mathcal{U}_{1} \mathcal{G}_{m}^{(2)} \mathcal{U}_{1}^{-1} & =\mathcal{Q}_{m}^{(1)}
\end{aligned}
$$

where (1) and (2) denote the topological generators. They are obtained through the twistings (1) and (2) of the superconformal generators, namely

$$
\begin{aligned}
\mathcal{L}_{m}^{(1)} & =L_{m}+\frac{1}{2}(m+1) H_{m}, \\
\mathcal{H}_{m}^{(1)} & =H_{m}, \\
\mathcal{G}_{m}^{(1)} & =G_{m+\frac{1}{2}}^{+}, \quad \mathcal{Q}_{m}^{(1)}=G_{m-\frac{1}{2}}^{-}
\end{aligned}
$$

and

$$
\begin{aligned}
\mathcal{L}_{m}^{(2)} & =L_{m}-\frac{1}{2}(m+1) H_{m}, \\
\mathcal{H}_{m}^{(2)} & =-H_{m}, \\
\mathcal{G}_{m}^{(2)} & =G_{m+\frac{1}{2}}^{-}, \quad \mathcal{Q}_{m}^{(2)}=G_{m-\frac{1}{2}}^{+},
\end{aligned}
$$

and $\mathcal{U}_{1}$ is the spectral flow operator $\mathcal{U}_{\theta}$ for $\theta=1$ (one gets the same transformation (1.1) exchanging $(1) \leftrightarrow(2)$ and $\left.\mathcal{U}_{1} \leftrightarrow \mathcal{U}_{-1}\right)$.

Observe that the topological chiral primaries, i.e. those annihilated by $\mathcal{Q}_{0}$ and $\mathcal{G}_{0}$, are the superconformal chiral primaries belonging to the chiral ring and the antichiral ring, for the twists (2) and (1) respectively.

In ref. [1] we argued that, as long as we stay at the topological algebra level without going into specific realizations of the twisted topological theories, we can regard (1.1), without labels (1) and (2), as a topological algebra automorphism. The reason is that both sets of generators satisfy exactly the same algebra, so that at the topological algebra level they are indistinguishable from each other. This topological algebra automorphism was expressed introducing a new operator $\mathcal{A}$ in the form 


$$
\begin{aligned}
\mathcal{A} \mathcal{L}_{m} \mathcal{A} & =\mathcal{L}_{m}-m \mathcal{H}_{m} \\
\mathcal{A} \mathcal{H}_{m} \mathcal{A} & =-\mathcal{H}_{m}-\frac{\mathrm{c}}{3} \delta_{m, 0} \\
\mathcal{A} \mathcal{Q}_{m} \mathcal{A} & =\mathcal{G}_{m} \\
\mathcal{A} \mathcal{G}_{m} \mathcal{A} & =\mathcal{Q}_{m}
\end{aligned}
$$

with $\mathcal{A}^{-1}=\mathcal{A}$.

In this note we intend to bring to the reader's attention the fact that the untwisting of the topological algebra automorphism (1.4) does not give back the "usual" spectral flow on the $\mathrm{N}=2$ superconformal generators, but a different transformation: the "other"

spectral flow. In what follows we will discuss the similarities and differences between the two versions of the spectral flow.

\section{The Usual Spectral Flow}

Let us start with the usual spectral flow [2], [3]. It is given by the one-parameter family of transformations

$$
\begin{aligned}
\mathcal{U}_{\theta} L_{m} \mathcal{U}_{\theta}^{-1} & =L_{m}+\theta H_{m}+\frac{c}{6} \theta^{2} \delta_{m, 0}, \\
\mathcal{U}_{\theta} H_{m} \mathcal{U}_{\theta}^{-1} & =H_{m}+\frac{c}{3} \theta \delta_{m, 0}, \\
\mathcal{U}_{\theta} G_{r}^{+} \mathcal{U}_{\theta}^{-1} & =G_{r+\theta}^{+}, \\
\mathcal{U}_{\theta} G_{r}^{-} \mathcal{U}_{\theta}^{-1} & =G_{r-\theta}^{-} .
\end{aligned}
$$

on the generators of the $\mathrm{N}=2$ superconformal algebra 四, [5], satisfying $\mathcal{U}_{\theta}^{-1}=\mathcal{U}_{(-\theta)}$. These transformations give rise to isomorphic algebras.

In order to simplify the analysis that follows, it will be very convenient to unify the notation for the $U(1)$ charge of the states of the Neveu-Schwarz (aperiodic) algebra and the states of the Ramond (periodic) algebra. Namely, the $U(1)$ charge of the Ramond states will be denoted by $h$, instead of $h \pm \frac{1}{2}$. In addition, the relative charge of a secondary state $q$ will be defined as the difference between the $U(1)$ charge of the state and the $U(1)$ charge of the primary on which it is built. Therefore, the relative charges of the Ramond states are defined to be integer (in contrast with the usual definition).

Let us denote by $(\Delta, h)$ the $\left(L_{0}, H_{0}\right)$ eigenvalues of any given state $|\chi\rangle$, then it is easy to see that the eigenvalues of the transformed state $\mathcal{U}_{\theta}|\chi\rangle$ are $\left(\Delta-\theta h+\frac{c}{6} \theta^{2}, h-\frac{c}{3} \theta\right)$. If the state $|\chi\rangle$ is a level- $l$ secondary state with relative charge $q$ and eigenvalues $(\Delta+l, h+q)$ (where now $(\Delta, h)$ denote the eigenvalues of the primary on which the secondary is built), then one gets straightforwardly that the level of the transformed state $\mathcal{U}_{\theta}|\chi\rangle$ changes to $l-\theta q$, while the relative charge remains equal. 
In the discussion that follows we will assume the same behaviour for left-movers and right-movers, so that no distinction between them will be made.

For half-integer values of $\theta$ the spectral flow (2.1) interpolates between the NS algebra and the $\mathrm{R}$ algebra. In particular, for $\theta=\frac{1}{2}$ the primaries of the NS algebra become primaries of the $\mathrm{R}$ algebra, with chirality $(-)$ (i.e. annihilated by $G_{0}^{-}$), and the chiral ring (the primaries of the NS algebra annihilated by $G_{-1 / 2}^{+}$) is mapped into the set of Ramond ground states (annihilated by both $G_{0}^{+}$and $G_{0}^{-}$). Similarly, for $\theta=-\frac{1}{2}$ the primaries of the NS algebra are transformed into primaries of the $\mathrm{R}$ algebra with chirality $(+)$ and the antichiral ring (primaries annihilated by $G_{-1 / 2}^{-}$) is mapped into the Ramond ground states.

For integer values of $\theta$ the $\mathrm{N}=2$ superconformal algebras map back to themselves, although the primary fields change, i.e. the fields that were primary with respect to the initial algebra are in general not longer primary with respect to the final algebra. Only in particular cases the spectral flow maps the initial primary states into primary states as well. For example, in the case of the NS algebra only primary states that are chiral (or antichiral) can be mapped into primary states of the NS algebra. In particular, for $\theta=1$ the chiral ring is mapped into the antichiral ring (while the antichiral ring is mapped to a set of non-primary fields), and for $\theta=-1$ the antichiral ring is mapped into the chiral ring (while the chiral ring is mapped into non-primary fields).

In the case of the $\mathrm{R}$ algebra, the spectral flow with $\theta=1$ transforms primary states with chirality $(+)$ into primary states with chirality $(-)$, while primary states with chirality $(-)$ become non-primaries and annihilated by $G_{-1}^{-}$. Therefore, the Ramond ground states are transformed under $\mathcal{U}_{1}$ into chirality $(-)$ primaries with the additional condition of being annihilated by $G_{-1}^{-}$. Similarly, for $\theta=-1$ the primary states with chirality (-) are transformed into primary states with chirality $(+)$ and the ground states are mapped into chirality $(+)$ primaries with the additional condition of being annihilated by $G_{-1}^{+}$.

\section{The Other Spectral Flow}

The untwisting of the topological algebra automorphism (1.4), under the twist (1), eq. (1.2), gives the transformation

$$
\begin{aligned}
\mathcal{A}^{(1)} L_{m} \mathcal{A}^{(1)} & =L_{m}+H_{m}+\frac{\mathrm{c}}{6} \delta_{m, 0} \\
\mathcal{A}^{(1)} H_{m} \mathcal{A}^{(1)} & =-H_{m}-\frac{\mathrm{c}}{3} \delta_{m, 0} \\
\mathcal{A}^{(1)} G_{r}^{+} \mathcal{A}^{(1)} & =G_{r-1}^{-} \\
\mathcal{A}^{(1)} G_{r}^{-} \mathcal{A}^{(1)} & =G_{r+1}^{+}
\end{aligned}
$$


while under the twist (2), eq. (1.3), it results in

$$
\begin{aligned}
\mathcal{A}^{(2)} L_{m} \mathcal{A}^{(2)} & =L_{m}-H_{m}+\frac{\mathrm{c}}{6} \delta_{m, 0}, \\
\mathcal{A}^{(2)} H_{m} \mathcal{A}^{(2)} & =-H_{m}+\frac{\mathrm{c}}{3} \delta_{m, 0} \\
\mathcal{A}^{(2)} G_{r}^{+} \mathcal{A}^{(2)} & =G_{r+1}^{-} \\
\mathcal{A}^{(2)} G_{r}^{-} \mathcal{A}^{(2)} & =G_{r-1}^{+}
\end{aligned}
$$

where $\mathcal{A}^{(1)}$ and $\mathcal{A}^{(2)}$ denote the "untwisted" automorphisms under the twists (1) and (2) respectively.

One can therefore regard the transformations (3.1) and (3.2) as the particular cases, for $\theta=1$ and $\theta=-1$ respectively, of a different spectral flow given by

$$
\begin{aligned}
\mathcal{A}_{\theta} L_{m} \mathcal{A}_{\theta} & =L_{m}+\theta H_{m}+\frac{\mathrm{c}}{6} \theta^{2} \delta_{m, 0}, \\
\mathcal{A}_{\theta} H_{m} \mathcal{A}_{\theta} & =-H_{m}-\frac{\mathrm{c}}{3} \theta \delta_{m, 0}, \\
\mathcal{A}_{\theta} G_{r}^{+} \mathcal{A}_{\theta} & =G_{r-\theta}^{-} \\
\mathcal{A}_{\theta} G_{r}^{-} \mathcal{A}_{\theta} & =G_{r+\theta}^{+} .
\end{aligned}
$$

Observe that $\mathcal{U}_{\theta}^{-1}=\mathcal{U}_{(-\theta)}$ while $\mathcal{A}_{\theta}^{-1}=\mathcal{A}_{\theta}$, i.e. the inverse of the usual spectral flow with parameter $\theta$ is the one given by $(-\theta)$ while for the other spectral flow the transformation and its inverse coincide. The $\left(L_{0}, H_{0}\right)$ eigenvalues of the transformed states $\mathcal{A}_{\theta}|\chi\rangle$ are now $\left(\Delta+\theta h+\frac{c}{6} \theta^{2},-h-\frac{c}{3} \theta\right)$ (that is, they differ from the previous case by the interchange $h \rightarrow-h$ ). From this one easily deduces that, under the spectral flow (3.3), the level $l$ of any descendant will change to $l+\theta q$ while the relative charge $q$ reverse its sign.

Observe also that, due to the untwisting procedure, the natural Hilbert space of the transformation given by (3.1) is the antichiral ring, while the natural Hilbert space of the transformation given by (3.2) is the chiral ring. As a matter of fact, (3.1) maps the antichiral ring into the antichiral ring (transforming the chiral ring into a set of nonprimary fields), while (3.2) maps the chiral ring into the chiral ring (and the antichiral ring into non-primary fields). Not only that, but for no values of $\theta$ does the spectral flow (3.3) interpolate between the chiral ring and the antichiral ring. In addition, as happened with the usual spectral flow, only primaries of the NS algebra that are chiral or antichiral can be mapped into primaries of the NS algebra.

For half-integer values of $\theta$ the spectral flow (3.3) also interpolates between the NS algebra and the $\mathrm{R}$ algebra. For $\theta=\frac{1}{2}$ the primaries of the NS algebra become primaries of the $\mathrm{R}$ algebra with chirality $(-)$, as before, but now it is the antichiral ring which is mapped into the Ramond ground states. Similarly, for $\theta=-\frac{1}{2}$ the primaries of the NS algebra are transformed into primaries of the $\mathrm{R}$ algebra with chirality $(+)$, while now the chiral ring is mapped into the Ramond ground states. 
In the case of the $\mathrm{R}$ algebra, the spectral flow (3.3) with $\theta=1$ transforms primary states with chirality $(-)$ back into chirality $(-)$ primaries; that is, $\mathcal{A}_{1}$ does not reverse the chirality as $\mathcal{U}_{1}$ does. Primary states with chirality $(+)$, in turn, are transformed into nonprimary states annihilated by $G_{-1}^{-}$. Therefore, the Ramond ground states are transformed under $\mathcal{A}_{1}$ into chirality $(-)$ primaries with the additional condition of being annihilated by $G_{-1}^{-}$. Similarly, for $\theta=-1$ the primary states with chirality $(+)$ are transformed back into primary states with chirality $(+)$ and the ground states are mapped into chirality $(+)$ primaries with the additional condition of being annihilated by $G_{-1}^{+}$.

\section{Spectral Flows on Null States}

Now let us discuss the behaviour of the spectral flows (2.1) and (3.3) when they act on null states; that is, descendant states that also satisfy the highest weight conditions

$$
L_{n>0}|\chi\rangle=H_{n>0}|\chi\rangle=G_{n>0}^{+}|\chi\rangle=G_{n>0}^{-}|\chi\rangle=0
$$

which define primary states. Since null states are also primaries, we can apply the analysis above regarding the action of the spectral flows on primary states. However, we have to take into account that null states of the NS algebra cannot be chiral or antichiral. In particular, this applies to null states built on the chiral ring and the antichiral ring, i.e. they cannot be chiral or antichiral themselves (a detailed analysis of this fact will be given in [7]). Also we have to take into account that the possible values of the relative charge $q$ are very limited for null states: zero and \pm 1 [6] (in the usual notation $\pm \frac{1}{2}$ and $\pm \frac{3}{2}$ for the null vectors of the Ramond algebra).

The main features about the action of the spectral flows (2.1) and (3.3) on null states, as deduced from the above discussions, are therefore the following, where $|\chi\rangle_{l}^{(q)}$ denotes a level-l null state with relative charge $q$.

*) The spectral flows (2.1) and (3.3) map null states $|\chi\rangle_{l}^{(q)}$ of the NS algebra into null states of the $\mathrm{R}$ algebra (and viceversa), for $\theta= \pm \frac{1}{2}$. The $\left(L_{0}, H_{0}\right)$ eigenvalues of the primaries on which the $\mathrm{R}$ null states are built are given by $\left(\Delta \pm \frac{1}{2} h+\frac{c}{24}, h \pm \frac{c}{6}\right)$, using the usual spectral flow, and $\left(\Delta \pm \frac{1}{2} h+\frac{c}{24},-\left(h \pm \frac{c}{6}\right)\right)$, using the other spectral flow, where $(\Delta, h)$ are the eigenvalues of the primaries on which the NS null states are built. The levels of the $\mathrm{R}$ null states are given by $l \pm \frac{1}{2} q$, the sign depending on the sign of $\theta$ and the specific spectral flow, and the relative charges are given by $q$ (usual spectral flow) and $-q$ (other spectral flow). The chiralities of the $\mathrm{R}$ null states are $(-)$ for $\theta=\frac{1}{2}$ and $(+)$ for $\theta=-\frac{1}{2}$. 
*) For no value of $\theta$ null states of the NS algebra are mapped into null states of the NS algebra. This applies in particular to null states built on the chiral ring and the antichiral ring.

*) Null states of the $\mathrm{R}$ algebra are mapped into null states of the $\mathrm{R}$ algebra, for $\theta= \pm 1$. The $\left(L_{0}, H_{0}\right)$ eigenvalues of the primaries on which the null states are built change from $(\Delta, h)$ to $\left(\Delta \pm h+\frac{c}{6}, h \pm \frac{c}{3}\right)$, using the usual spectral flow, and to $\left(\Delta \pm h+\frac{c}{6},-\left(h \pm \frac{c}{3}\right)\right)$, using the other spectral flow. The level of the null states gets modified to $l \pm q$, the sign depending on the sign of $\theta$ and the spectral flow at hand, while only the other spectral flow modifies the relative charge, reversing its sign. In addition, the usual spectral flow reverses the chirality, while the other spectral flow does not.

\section{Final Remarks}

We have shown that the "usual" spectral flow $\mathcal{U}_{\theta}$ and the "other" spectral flow $\mathcal{A}_{\theta}$, although similar in many of their properties, differ notably in some respects. Their behaviour with respect to the chiral ring and the antichiral ring is drastically different, and complementary. The usual spectral flow interpolates between the chiral ring and the antichiral ring, but does not connect the chiral ring and the antichiral ring back to themselves. The other spectral flow does exactly the opposite, mapping the chiral ring and the antichiral ring back to themselves, while being unable to connect the two rings to each other. Furthermore their inverses are different : $\mathcal{U}_{\theta}^{-1}=\mathcal{U}_{(-\theta)}$ and $\mathcal{A}_{\theta}^{-1}=\mathcal{A}_{\theta}$.

We have already found a practical application of this result. As we discussed above, the spectral flows themselves do not map null states of the NS algebra into null states of the NS algebra. However, using the two spectral flows we have constructed operators that map null states of the NS algebra into null states of the NS algebra [7], [8]. In particular, using the usual spectral flow, the resulting operators transform null states built on the chiral ring into null states built on the antichiral ring (and viceversa). On the other hand, using the other spectral flow, the corresponding operators map null states built on the chiral ring into null states built on the chiral ring, and null states built on the antichiral ring into null states built on the antichiral ring.

We think that other applications will be found for the "other" spectral flow described here.

\section{References}


[1] B. Gato-Rivera and J.I. Rosado, "Spectral Flows and Twisted Topological Theories", IMAFF-95/35 (1995), hep-th/9504056, to be published in Phys. Lett. B

[2] A. Schwimmer and N. Seiberg, Phys. Lett. B184 (1987) 191

[3] W. Lerche, C. Vafa and N. P. Warner, Nucl. Phys. B324 (1989) 427

[4] M. Ademollo et al., Phys. Lett. B62 (1976) 105; Nucl. Phys. B111 (1976) 77

[5] P. Di Vecchia et al., Phys. Lett. B162 (1985) 327

[6] W. Boucher, D. Friedan and A. Kent, Phys. Lett. B172 (1986) 316

[7] B. Gato-Rivera and J.I. Rosado, "Families of Singular States of the Twisted Topological and Untwisted N=2 Superconformal Algebras", to appear in hep-th

[8] B. Gato-Rivera and J.I. Rosado, "More Mappings between Singular States of the $\mathrm{N}=2$ Superconformal Algebra", to appear in hep-th 